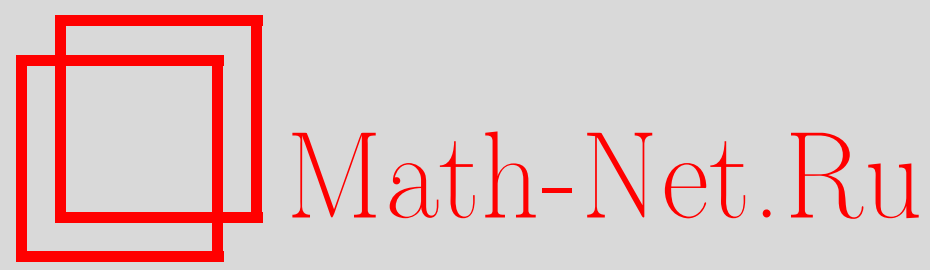

И. Э. Зверович, Характеризация хорошо укрытых графов в терминах запрещенных костабильных подграфов, $M a$ тем. заметки, 2000, том 67, выпуск 1, 52-56

DOI: https://doi.org/10.4213/mzm813

Использование Общероссийского математического портала Math-Net.Ru подразумевает, что вы прочитали и согласны с пользовательским соглашением http://www . mathnet.ru/rus/agreement

Параметры загрузки:

IP: 54.172 .240 .79

26 апреля 2023 г., 14:58:45

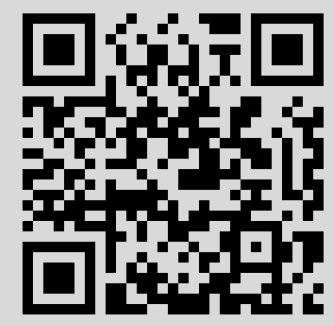




\section{ХАРАКТЕРИЗАЦИЯ ХОРОШО УКРЫТЫХ ГРАФОВ В ТЕРМИНАХ ЗАПРЕЩЕННЫХ КОСТАБИЛЬНЫХ ПОДГРАФОВ}

И.Э. Зверович

Граф̆ называется хорошо укрытым, если каждое максимальное независимое множество является наибольшим. Пусть $I$ - независимое множество (возможно, пустое) в графе $G$. Подграф графа $G$, полученный удалением множества $I$ вместе с окрестностью, называется костабильным.

Получена характеризация класса хорошо укрытых графов в терминах минимального множества запрещенных костабильных подграбов. Из нее вытекают характеризации известных подклассов класса хорошо укрытых графов и существование полиномиального алгоритма распознавания хорошо укрытых графов с ограниченньми степенями вершин.

Библиограффия: 8 названий.

Мы используем терминологию книги [1]. Классомграфов назьвается любое множество графов, различаемых с точностью до изоморфизма. Пусть $\operatorname{Ind}(G)$ есть множество всех максимальных независимых множеств графа $G ; \alpha(G)=\max \{|I|: I \in \operatorname{Ind}(G)\}-$ иисло независимости графа $G$. Граф $G$ называется хорошо укрытым, если $|I|=\alpha(G)$ для любого $I \in \operatorname{Ind}(G)$. Обозначим через $W$ класс всех хорошо укрытых графов. В [2] и [3] доказано, что задача распознавания класса $W$ является со-NP-полной. Имеется обзор [4] по хорошо укрытым графам. Основные проблемы в рассматриваемой области заключаются в выявлении полиномиально распознаваемых классов хорошо укрытых графов и их характеризации.

Через $N(x)$ обозначается окрестность вершины $x$. Для множества $X \subseteq V G$ обозначим $N(X)=\bigcup_{x \in X} N(x)$ и $N[X]=X \cup N(X)$. Пусть $I$ - независимое множество (возможно, пустое) в графе $G$. Если $I \notin \operatorname{Ind}(G)$, то подграф $G-N[I]$ называется костабильным. Обозначим через $\operatorname{CSub}(G)$ множество всех костабильных подграфов графа $G$. Класс графов $P$ назовем конаследственным, если $\operatorname{CSub}(G) \subseteq P$ для любого графа $G \in P$.

ПРЕДЛОЖЕНИЕ 1. Класс $W$ является конаследственным.

ДокАЗАтЕЛЬСтво. Пусть $G \in W$ и $H \in \operatorname{CSub}(G)$. По определению $H=G-$ $N[I]$, где $I$ - независимое множество в $G$. Пусть $J, J^{\prime} \in \operatorname{Ind}(H)$. Ясно, что $J \cup I$, $J^{\prime} \cup I \in \operatorname{Ind}(G)$. Так как $G \in W$, то $|J \cup I|=\left|J^{\prime} \cup I\right|$, откуда $|J|=\left|J^{\prime}\right|$. Поэтому $H \in W$.

Для множества графов $Z$ положим

$$
\operatorname{FCS}(Z)=\{G: \operatorname{CSub}(G) \cap Z=\varnothing\} .
$$


Если $P=\mathrm{FCS}(Z)$, то $Z$ назьвается множеством запрещенных костабильных подграфов для $P$.

Запрещенньй костабильньй подграф $H \in Z$ для класса $P$ называется минимальным, если $\operatorname{CSub}(H) \backslash\{H\} \subseteq P$.

ПРЕДЛОЖЕНИЕ 2. і) Класс $Р$ является конаследственным тогда и только тогда, когда $P=\operatorname{FCS}(Z)$ для некоторого множества графов $Z$.

ii) Минимальное (по включению) мнохество $Z$, удовлетворяющее і), определено однозначно.

ДокаЗАтельство. і) Необходимость. Пусть $P$ является конаследственным классом и $G \in P$. Положим $Z=\{H: H \notin P\}$. Так как $P$ - конаследственньй класс, то $\operatorname{CSub}(G) \subseteq P$. Следовательно, $\operatorname{CSub}(G) \cap Z=\varnothing$, т.е. $G \in \operatorname{FCS}(Z)$ и $P \subseteq \operatorname{FCS}(Z)$. Обратно, пусть $G \in \operatorname{FCS}(Z)$, т.е. $\operatorname{CSub}(G) \cap Z=\varnothing$. Так как $G \in \operatorname{CSub}(G)$, то $G \notin Z$. Следовательно, $G \in P$ и $\operatorname{FCS}(Z) \subseteq P$.

Достаточность следует из того, что $\operatorname{CSub}(H) \subseteq \operatorname{CSub}(G)$, если $H \in \operatorname{CSub}(G)$.

ii) Пусть $P=\operatorname{FCS}(Z)$ и $Z^{o}$ есть множество всех минимальных костабильных подграфов для $P$. Достаточно показать, что $Z^{o} \subseteq Z$ и $P=\operatorname{FCS}\left(Z^{o}\right)$.

Пусть $G \in Z^{o}$. Тогда $G \notin P=\operatorname{FCS}(Z)$, откуда $\operatorname{CSub}(G) \cap Z \neq \varnothing$. Из минимальности $G$ следует, что $\operatorname{CSub}(G) \backslash\{G\} \subseteq P$. Кроме того, очевидно, что $Z \cap P=\varnothing$. Таким образом, $\operatorname{CSub}(G) \cap Z=\{G\}$ и $G \in Z$. Итак, $Z^{o} \subseteq Z$.

Так как $Z^{o} \cap P=\varnothing$, то $P \subseteq \operatorname{FCS}\left(Z^{o}\right)$.

Обратно, пусть $G \in \operatorname{FCS}\left(Z^{o}\right)$, т.е. $\operatorname{CSub}(G) \cap Z^{o}=\varnothing$. Допустим, что $G \notin P$. Из конечности множества $\operatorname{CSub}(G)$ следует, что существует граф $H \in \operatorname{CSub}(G)$ такой, что $H \notin P$ и $\operatorname{CSub}(H) \backslash\{H\} \subseteq P$, т.е. $H \in Z^{o}$. Противоречие с $\operatorname{CSub}(G) \cap Z^{o}=\varnothing$. Поэтому $G \in P$ и $\operatorname{FCS}\left(Z^{o}\right) \subseteq P$.

Соединением графов $G_{1}, G_{2}, \ldots, G_{k}$ (при условии $V G_{i} \cap V G_{j}=\varnothing$, где $1 \leqslant i \neq$ $j \leqslant k$ ) назьвается граф $\sum_{i=1}^{k} G_{i}=\overline{\overline{G_{1}} \cup \overline{G_{2}} \cup \cdots \cup \overline{G_{k}}}$. Соединение двух графов $G$ и $H$ обозначается $G+H$. Положим

$$
\begin{aligned}
Z_{W}= & \left\{\sum_{i=1}^{k} G_{i}: k \geqslant 2, G_{i} \in W \text { при } i=1,2, \ldots, k\right. \text { и } \\
& \left.\alpha\left(G_{i}\right) \neq \alpha\left(G_{j}\right) \text { при } 1 \leqslant i \neq j \leqslant k\right\} .
\end{aligned}
$$

Tеорема 1. $W=\operatorname{FCS}\left(Z_{W}\right)$, причем $Z_{W}$ является множеством минимальных запрещенных костабильных подграфов для $W$.

ДокАЗАТЕЛьство. Легко видеть, что ни одинграф множества $Z_{W}$ не является хорошо укрытым. В силу конаследственности класса $W$ (предложение 1) имеем $W \subseteq \operatorname{FCS}\left(Z_{W}\right)$.

Для доказательства обратного включения рассмотрим минимальньй граф $G \notin W$.

Лемма 1. Если $I, J \in \operatorname{Ind}(G) u|I| \neq|J|$, mo $I \cap J=\varnothing$. 
ДоКАЗАТЕЛЬСтво. Несложно видеть, что множества $I_{1}=I \backslash J$ и $J_{1}=J \backslash I$ являются максимальными независимьги в подграфе $G_{1}=G-N[I \cap J]$, причем $\left|I_{1}\right| \neq\left|J_{1}\right|$. Следовательно, $G_{1} \notin W$. Так как $G_{1} \in \operatorname{CSub}(G)$, в силу минимальности графа $G$ имеем $G=G_{1}$, т.е. $I \cap J=\varnothing$.

Продолжим доказательство теоремы 1 . По лемме 1 любые два максимальные независимые множества, содержащие вершину $u$ графа $G$, равномощны. Обозначим через $i(u)$ мощность максимального независимого множества, содержащего вершину $u$. На множестве $V G$ определим отношение эквивалентности: $u R v$ тогда и только тогда, когда $i(u)=i(v)$. Пусть $C_{1}, C_{2}, \ldots, C_{k}-$ классы эквивалентности, определяемые отношением $R$. Так как $G \notin W$, то $k \geqslant 2$.

Допустим, что существуют несмежные вершины $x \in C_{i}$ и $y \in C_{j}(1 \leqslant i \neq j \leqslant k)$. Выберем произвольное множество $I \in \operatorname{Ind}(G)$, содержащеее $x$ и $y$. Так как $x \in I$ и $y \in I$, то $|I|=i(x)$ и $|I|=i(y)$. Но $i(x) \neq i(y)$, поскольку $i \neq j$. Противоречие. Поэтому $G=\sum_{i=1}^{k} G_{i}$, где $G_{i}=G\left(C_{i}\right), i=1,2, \ldots, k$.

Отсюда следует, что $\operatorname{Ind}(G)=\bigcup_{i=1}^{k} \operatorname{Ind}\left(G_{i}\right)$ и $\operatorname{Ind}\left(G_{i}\right) \cap \operatorname{Ind}\left(G_{j}\right)=\varnothing$ при $1 \leqslant i \neq$ $j \leqslant k$. Из определения $C_{i}$ вытекает, что $G_{i} \in W$. Имеем $G \in Z_{W}$. Следовательно, $\operatorname{FCS}\left(Z_{W}\right) \subseteq W$. Равенство $W=\operatorname{FCS}\left(Z_{W}\right)$ доказано.

Покажем, что $Z_{W}$ является множеством минимальных запрешенных костабильных подграфов. Пусть $G=\sum_{i=1}^{k} G_{i} \in Z_{W}$, где $k \geqslant 2$ и $G_{i} \in W$. Пусть $I \neq \varnothing-$ независимое множество в $G$. Ясно, что $I \subseteq V G_{i}$ при некотором $i \in\{1,2, \ldots, k\}$. Тогда получаем, что $G-N[I]=G_{i}-N[I] \in W$, поскольку $G_{i} \in W$ и по предложению 1 класс $W$ является конаследственным.

В ряде работ (например, [4]-[7]) рассматриваются некоторые наследственные подклассы класса $W$, определяемые запрешенными порожденньми подграфами типа $C_{3}$, $C_{4}, K_{1,3}, K_{1,4}$ или их комбинациями. Из теоремы 1 легко получить характеризации таких подклассов в терминах запрещенных костабильных подграфов.

Напомним, что обхватом $g(G)$ графа $G$ называется длина кратчайшего простого цикла в $G$.

СлЕдСтВие 1. і) $E c л и ~ g(G) \geqslant 4$, mo $G \in W$ тогда и только тогда, когда $G$ не содержит полных двудольных графов с неравномощными долями в качестве костабильных подграфов.

ii) $\operatorname{Ecли~} g(G) \geqslant 5$, то $G \in W$ тогда и только тогда, когда $G$ не содержит звезд порядка по крайней мере 3 в качестве костабильных подграфов.

iii) Если граф $G$ не содержит порохденного подграфа $K_{1,3}$, то $G \in W$ тогда и только тогда, когда $G$ не содержит графов вида $K_{n}+H$, где $n \geqslant 1$ ( $n$ не фиксировано) и $H \in W, \alpha(H)=2$, в качестве костабильных подграфов.

iv) $E с л и ~ G$ не содержит порожденного подграфа $C_{4}$, то $G \in W$ тогда и только тогда, когда $G$ не содержит графов вида $K_{n}+H$, где $n \geqslant 1$ ( $n$ не фиксировано) $u$ $H \in W, \alpha(H) \geqslant 2$, в качестве костабильных подграфов.

ДокАЗАТЕЛьСтво. Легко видеть, что если граф $G$ не содержит граф $H$ в качестве порожденного подграфа, то любой костабильньй подграф графа $G$ также не содержит $H$ в качестве порожденного подграфа. По теореме 1 нам достаточно удалить из $Z_{W}$ все графы, содержащие запрещенные порожденные подграфы в каждом случае i) - iv).

Пусть $G=\sum_{i=1}^{k} G_{i} \in Z_{W}\left(k \geqslant 2, G_{i} \in W, \alpha\left(G_{i}\right) \neq \alpha\left(G_{j}\right)\right.$ при $\left.i \neq j\right)$. 
i) Класс графов $\{G: g(G) \geqslant 4\}$ определяется запрещенным порожденным подграфом $C_{3}$. Если $G$ не содержит $C_{3}$, то $k=2$ и все графы $G_{i}$ пустые, т.е. $G$ является полным двудольным графом с неравномощными долями.

ii) Класс графов $\{G: g(G) \geqslant 5\}$ определяется запрешенными порожденными подграфами $C_{3}$ и $C_{4}$. Так как $C_{3}$ запрещен, то граф $G$ такой, как в і). Из отсутствия порожденного подграфа $C_{4}$ следует, что $G$ является звездой $K_{1, n}$ с $n \geqslant 2\left(\right.$ так как $\left.\alpha\left(G_{i}\right) \neq \alpha\left(G_{j}\right)\right)$.

iii) Если граф $G$ не содержит порожденного подграфа $K_{1,3}$, то $\alpha\left(G_{i}\right) \leqslant 2$ для любого $i=1,2, \ldots, k$. Но $\alpha\left(G_{i}\right) \neq \alpha\left(G_{j}\right)$ при $i \neq j$. Поэтому $k=2, \alpha\left(G_{1}\right)=1$ и $\alpha\left(G_{2}\right)=2$.

iv) Если $G$ не содержит порожденного подграфа $C_{4}$, то все $G_{i}$, кроме одного, являются полными графами. Так как $\alpha\left(G_{i}\right) \neq \alpha\left(G_{j}\right)$, то $k=2, G_{1}-$ полньй граф и $G_{2} \in W$, причем $\alpha\left(G_{2}\right) \geqslant 2$.

Проблема распознавания класса $W$ рассматривается также для грабов с ограниченными степенями вершин. Так, в [8] решены задачи характеризации и полиномиального распознавания 4-регулярных 4 -связных $K_{1,3}$-свободных хорошо укрытых графов. Мы покажем ниже, что сушествует полиномиальный алгоритм распознавания хорошо укрытых графов с ограниченными степенями вершин. Обозначим через $\Delta(G)$ максимальную степень вершин графа $G$.

СлЕДСТВИЕ 2. При любом $d \geqslant 0$ существует конечное множсество графов $Z(d)$ такое, что если $\Delta(G) \leqslant d$, то $G \in W$ тогда и только тогда, когда $G \in \operatorname{FCS}(Z(d))$.

ДокАЗАТЕЛЬСТво. По теореме 1 достаточно показать, что порядок любого графа $H \in Z_{W} \cap\{G: \Delta(G) \leqslant d\}$ ограничен. Пусть $H=\sum_{i=1}^{k} H_{i}$, где $k \geqslant 2$ и $H_{i} \in W$.

Каждая вершина $x \in V H_{1}$ смежна с каждой вершиной множества $V H \backslash V H_{1}$. Поэтому $d \geqslant \Delta(H) \geqslant \operatorname{deg}_{H} x \geqslant\left|V H \backslash V H_{1}\right|$. Аналогично, $d \geqslant\left|V H \backslash V H_{2}\right|$. Тогда $2 d \geqslant\left|V H \backslash V H_{1}\right|+\left|V H \backslash V H_{2}\right| \geqslant|V H|$.

ПРЕДЛОЖЕНИЕ 3. Если $P=\operatorname{FCS}(Z)$ и множество $Z$ конечно, то для любого $d \geqslant 0$ существует полиномиальный алгоритм распознавания класса $P \cap$ $\{G: \Delta(G) \leqslant d\}$.

ДокАЗАтЕЛьство. Пусть дан граф $G$ с $\Delta(G) \leqslant d$. Так как $Z$ конечно, порядок любого графа $H \in Z$ ограничен сверху. Поэтому заполиномиальноевремя можно найти все порожденные подграфы графа $G$, изоморфные $H$. Для любого множества $U \subseteq V G$ такого, что $G(U)=H$, рассмотрим множество $V=N(U) \backslash U$. Пусть $X=V G \backslash(U \cup V)$. Легко видеть, что подграф $G(U)$ является костабильным тогда и только тогда, когда существует такое независимое множество $I \subseteq X$, что $V \subseteq N(I)$ ( $I$ доминирует $V$ ). Мощность множества $V$ ограничена сверху: $|V| \leqslant d|U|$. Обозначим $V^{\prime}=(N(V) \backslash V) \cap$ $X$. Так как $\left|V^{\prime}\right| \leqslant d|V|$, за полиномиальное время можно построить все независимые множества $I^{\prime} \subseteq V^{\prime}$. Если существует $I^{\prime}$, которое доминирует $V$, то дополним $I^{\prime}$ до максимального независимого множества $I$ подграфа $G(X)$ и получим $H=G(U)=G-$ $N[I]$, т.е. $H \in \operatorname{CSub}(G)$.

Если указанного $I^{\prime}$ не существует, то $G(U) \notin \operatorname{CSub}(G)$.

СлЕДСТВИЕ 3. При любом $d \geqslant 0$ существует полиномиальный алгоритм распознавания класса $W \cap\{G: \Delta(G) \leqslant d\}$.

ДокАЗАТЕЛЬСТво вытекает из следствия 2 и предложения 3. 


\section{СПИСОК ЦИТИРОВАННОЙ ЛИТЕРАТУРЫ}

[1] Харари $\Phi$. Теория графово. М.: Мир, 1969.

[2] Chvátal V., Slater P. A note on well-covered graphs // Quo Vadis Graph Theory? (J. Gimbel et al., eds. ) Annals Discrete Math. (North-Holland, Amsterdam). 1993. V. 55. P. 179-182.

[3] Sankaranarayana R.S., Stewart L.K. Complexity results for well-covered graphs // Networks. 1992. V. 22. P. 247-262.

[4] Plummer M. D. Well-covered graphs: a survey // Questiones Math. 1993. V. 16. P. 253-287.

[5] Finbow A., Hartnell B., Nowakowski R. J. A characterization of well-covered graphs of girth 5 or greater // J. Combin. Theory Ser. B. 1993. V. 57. P. 44-68.

[6] Finbow A., Hartnell B., Nowakowski R. J. A characterization of well-covered graphs which contain neither 4- nor 5-cycles // J. Graph Theory. 1994. V. 18. P. 713-721.

[7] Whitehead C. A. A characterization of well-covered claw-free graphs containing no 4-cycle // Ars Combin. (to appear).

[8] Hartnell B., Plummer M. D. On 4-connected claw-free well-covered graphs // Discrete Appl. Math. 1996. V. 64. P. 57-65.

Белорусский государственный университет, г. Минск

Поступило

E-mail: igor@mmf.bsu.unibel.by

Исправленный вариант

15.04.1999 